BNL- -48478

DE93 006675

\title{
Study on the Results from SYNCH and MAD Programs in Calculating Lattice with Coordinate Rotation
}

\author{
Jianming Xu
}

December 1992

\section{DISCLAIMER}

This report was prepared as an account of work sponsored by an agency of the United States Government. Neither the United States Government nor any agency thereof, nor any of their employees, makes any warranty, express or implied, or assumes any legal liability or responsibility for the accuracy, completeness, or usefulness of any information, apparatus, product, or process disclosed, or represents that its use would not infringe privately owned rights. Reference herein to any specific commercial product, process, or service by trade name, trademark, manufacturer, or otherwise does not necessarily constitute or imply its endorsement, recommendation, or favoring by the United States Government or any agency thereof. The views and opinions of authors expressed herein do not necessarily state or reflect those of the United States Government or any agency thereof.

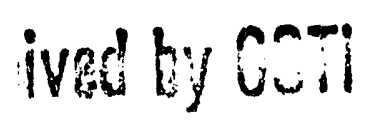

EB $O 21993$

\section{R H I C P R O J E T}

\author{
Brookhaven National Laboratory \\ Associated Universities, Inc. \\ Upton, NY 11973
}




\title{
Study on the Results from SYNCH and MAD Programs in Calculating Lattice with Coordinate Rotation
}

\author{
Jianming $\mathrm{Xu}$ \\ Brookhaven National Laboratory \\ December 1992
}

\section{Introduction}

In some lattices, it is necessary to introduce coordinate rotation around the beam axis, for example, in such a lattice, where the horizontal deflection and the change in level are mixed. The results from SYNCH and MAD programs in calculating such a lattice have been studied and some discrepancies have been found. The output of SYNCH is shown in Table 1 and outputs from MAD are shown in Table 2 and Table 3. The arrangement of this simple lattice is shown in these outputs. The dispersion functions are discussed first and then the Twiss Parameters ( $\beta$ 's and $\alpha$ 's).

\section{Dispersion Functions}

1. Dispersion functions before coordinate rotation at $L_{32}$.

The dispersion functions before rotation $\eta_{x 0}, \eta_{x 0}^{\prime}, \eta_{y 0}, \eta_{y 0}^{\prime}$ from the outputs of

MAD and SYNCH are shown as:

MAD output (Table 2 and 3 )

$$
\begin{aligned}
& \eta_{x 0}=1.146593631, \quad \eta_{x 0}^{\prime}=0.02084258749 \\
& \eta_{y 0}=0.08803616920, \quad \eta_{y 0}^{\prime}=0.01253036558 .
\end{aligned}
$$

SYNCH TRKB ('Track Betatron Function) Output (Table 1)

$$
\begin{aligned}
& \eta_{x 0}=1.146593, \quad \eta_{x 0}^{\prime}=0.020843 ; \\
& \eta_{y 0}=0.088036, \quad \eta_{y 0}^{\prime}=0.012530 .
\end{aligned}
$$


SYNCH MMM (Matrix Multiplication) Output (Table 1, WST)

$$
\begin{aligned}
& m_{16}=\eta_{x 0}=1.14670914, \quad \eta_{x 0}^{\prime}=m_{26}=0.02083611 \\
& m_{36}=\eta_{y 0}=0.08803616, \quad m_{46}=\eta_{y 0}^{\prime}=0.01253036
\end{aligned}
$$

From above results, it is clear that without coordinate rotation, the dispersion functions from MAD and SYNCH TRKB output coincide with each other, but $\eta_{0 x}$ and $\eta_{0 x}^{\prime}$ from SYNCH MMM result differ little from both MAD and SYNCH TRKB outputs.

2. Dispersion functions after a rotation around the beam axis by an angle $\xi$ The relation between dispersion functions $\eta_{x}, \eta_{x}^{\prime}, \eta_{y}, \eta_{y}^{\prime}$ after rotation and $\eta_{x 0}, \eta_{x 0}^{\prime}, \eta_{y 0}$ and $\eta_{y 0}^{\prime}$ before rotation can be expressed as follows

$$
\begin{aligned}
& \eta_{x}=\eta_{x 0} \cos \xi+\eta_{y 0} \sin \xi \\
& \eta_{x}^{\prime}=\eta_{x 0}^{\prime} \cos \xi+\eta_{y 0}^{\prime} \sin \xi \\
& \eta_{y}=-\eta_{x 0} \sin \xi+\eta_{y 0} \cos \xi \\
& \eta_{y}^{\prime}=-\eta_{x 0}^{\prime} \sin \xi+\eta_{y 0}^{\prime} \cos \xi
\end{aligned}
$$

Putting Eq. (1), the MAD output before rotation into Eqs. (4)-(7), we get $(\xi=0.05 \mathrm{rad})$.

MAD by matrix multiplication

$$
\begin{aligned}
& \eta_{x}=1.149560662, \quad \eta_{x}^{\prime}=0.02144279695 \\
& \eta_{y}=0.0306203495, \quad \eta_{y}^{\prime}=0.01147301068
\end{aligned}
$$

The MAD outputs (from Tables 2 and 3 at $R_{1}$ ) are

$$
\begin{aligned}
& \eta_{x}=1.149560662, \quad \eta_{x}^{\prime}=0.02144279695 \\
& \eta_{y}=0.03062034973, \quad \eta_{y}^{\prime}=0.01147301068
\end{aligned}
$$

The MAD output (9) coincides with the result from matrix multiplication, Eq. (8).

Using the SYNCH TRKB output before rotation (Eq. (2)) as initial value by means of matrix multiplication Eqs. (4)-(7), we get 
SYNCH TRKB by matrix multiplication

$$
\begin{aligned}
& \eta_{x}=1.149560023, \quad \eta_{x}^{\prime}=0.02144319067 ; \\
& \eta_{y}=0.030620212, \quad \eta_{y}^{\prime}=0.01147262494 .
\end{aligned}
$$

But the SYNCH TRKB output after rotation at $R_{1}$ is thoroughly different. They are as follows (from Table 1)

$$
\begin{aligned}
& n_{x}=-1.102947, \quad \eta_{x}^{\prime}=-0.020049 \\
& \eta_{y}=-0.084685, \quad \eta_{y}^{\prime}=-0.012053 .
\end{aligned}
$$

The SYNCH TRKB output after coordinate rotation does not fulfill the coordinate rotation relationship, Eq. (4)- (7). It is wrong apparently.

The relation between the SYNCH MMM output before rotation [Eq. (3)] and after rotation (from Table 1, WSRT) fulfill the coordinate rotation relationship [Eqs. (4)-(7)]. The MMM output after rotation (Table 1, WSRT) is

$$
\begin{array}{ll}
\eta_{x}=1.14967602, & \eta_{x}^{\prime}=0.02143632 ; \\
\eta_{y}=0.03061457, \quad \eta_{y}^{\prime}=0.01147333 .
\end{array}
$$

The data in Eq. (12) differ from those in Eq. (9) (MAD output), because the data before rotation from SYNCH MMM output Eq. (3) differ from both SYNCH TRKB output Eq. (2) and MAD output Eq. (1).

3. Conclusion about dispersion function calculation.

For lattice without coordinate rotation, the MAD TWISS output coincides with SYNCH TRKB output but the SYNCH MMM output differs from them. Aiter coordinate rotation, the MAD TWISS output fulfills the coordinate rotation relationship but SYNCH TRKB output does not fulfill. The SYNCH MMM output after rotation fulfill the coordinate rotation relationship but its data differs from MAD output because its data before rotation differs from both MAD and SYNCH TRKB output. It seems that, the dispersion functions from MAD output are reliable. 


\section{Twiss Parameters $\beta, \alpha$}

1. We use the following initial conditions.

$$
\begin{aligned}
& \beta_{x i}=40.755237, \quad \alpha_{x i}=1.992883, \quad \gamma_{x i}=0.12198635 \\
& \beta_{y i}=16.997687, \quad \alpha_{y i}=-1.04618, \quad \gamma_{y i}=0.12322221 .
\end{aligned}
$$

Before rotation, the output of MAD and SYNCH TRKB (at L32) coincides with each other. They are as follows:

MAD output (from Table 2 and Table 3 )

$$
\begin{aligned}
& \beta_{x 0}=11.78884158, \quad \alpha_{x 0}=0.6635168614 ; \\
& \beta_{y 0}=54.09318824, \quad \alpha_{y 0}=-2.370235501 .
\end{aligned}
$$

SYNCH TRKB output (from Table 1)

$$
\begin{aligned}
& \beta_{x 0}=11.7888, \quad \alpha_{x 0}=0.663517 \\
& \beta_{y 0}=54.0932, \quad \alpha_{y 0}=-2.370235
\end{aligned}
$$

The initial Twiss parameter Eq. (13) and the final Twiss parameters Eq. (14) or (15) should fulfill the following relation

$$
\left(\begin{array}{l}
\beta_{0} \\
\alpha_{0} \\
\gamma_{0}
\end{array}\right)=\left(\begin{array}{ccc}
\mathrm{m}_{11}^{2} & -2 \mathrm{~m}_{11} \mathrm{~m}_{12} & \mathrm{~m}_{12}^{2} \\
-\mathrm{m}_{11} \mathrm{~m}_{21} & 1+2 \mathrm{~m}_{12} \mathrm{~m}_{21} & -\mathrm{m}_{12} \mathrm{~m}_{22} \\
\mathrm{~m}_{21}^{2} & -2 \mathrm{~m}_{21} \mathrm{~m}_{22} & \mathrm{~m}_{22}^{2}
\end{array}\right)\left(\begin{array}{l}
\beta_{i} \\
\alpha_{i} \\
\gamma_{i}
\end{array}\right)
$$

where $m$ 's are the transfer matrix elements. Using the corresponding $4 \times 4$ matrix from SYNCH MMM output (Table 1 WST) from Eqs. (13) and (16) we can calculate the $\beta$ 's, $\alpha$ 's at L32. The calculated results are shown as following which well coincide with the MAD and SYNCH TRKB output.

$$
\begin{aligned}
& \beta_{x 0}=11.78884092, \quad \alpha_{x 0}=0.66351697 \\
& \beta_{y 0}=54.09318664, \quad \alpha_{y 0}=-2.370235431 .
\end{aligned}
$$

The above result shows that without rotation the $\beta$ 's, $\alpha$ 's from MAD and SYNCH TRKB and the $4 \times 4$ transfer matrix from SYNCH MMM output are all reliable. 
2. The $4 \times 4$ transfer matrix after rotation from SYNCH MMM output (Table 2, WSRT) fulfill the coordinate rotation relation. The $4 \times 4$ matrix after rotation $(\xi=0.05)$ can be got by matrix multiplication, the result is shown as follows:

$$
M=\left(\begin{array}{cccc}
0.660723045 & 18.98334088 & -0.119785867 & 1.40630563 \\
-0.121925944 & -1.99336127 & -0.006135546 & 0.051179110 \\
-0.03306371 & -0.949958808 & -2.393720579 & 28.10267027 \\
0.00610138 & 0.099751203 & -0.122608639 & 1.022729245
\end{array}\right)
$$

The SYNCH MMM output is as follows (Table 1, WSRT)

$$
M=\left(\begin{array}{cccc}
0.66072304 & 18.98334088 & -0.11978587 & 1.40630563 \\
-0.12192595 & -1.99336127 & -0.00613555 & 0.05117912 \\
-0.03306371 & -0.94995881 & -2.39372058 & 28.10267027 \\
0.006160138 & 0.09975120 & -0.12260864 & 1.02272924
\end{array}\right)
$$

Eq. (19) well coincides with Eq. (18). It shows that the $4 \times 4$ matrix from SYNCH MMM output is reliable after rotation also.

3. The projection of the 4-dimensional emittance ellipsoid after rotation on $x x^{\prime}$ and $y y^{\prime}$ planes.

From the inverse transformation matrix after rotation (Table 1, IWSR) we can get the 4-dimensional emittance ellipsoid after rotation and then get its projection on $x x^{\prime}$ and $y y^{\prime}$ planes. They are expressed as follows:

$$
\begin{gathered}
0.120795313 x^{2}+2 \times 0.648550256 x x^{\prime}+11.76053439 x^{\prime 2}=1.0113922 \epsilon_{0} \\
0.120966214 y^{2}-2 \times 2.336044532 y y^{\prime}+53.3794012 y^{\prime 2}=1.0113922 \epsilon_{0}
\end{gathered}
$$

where $\epsilon_{0}$ is the initial $x$ and $y$ emittance. The projections depend upon the ratio of the initial $x$ and $y$ emittance. Here we take the ratio to be one. The corresponding $\beta, \alpha$ value of these projections are

$$
\begin{aligned}
& \beta_{x}=11.76053439, \quad \alpha_{x}=0.648550256 \\
& \beta_{y}=53.3794012, \quad \alpha_{y}=-2.336044532 .
\end{aligned}
$$


4. $\beta, \alpha$ values after rotation from $\mathrm{SYNCH}$ and $\mathrm{MAD}$ (at $R_{1}$ ) SYNCH TRKB output (Table 1)

$$
\begin{aligned}
& \beta_{x}=10.9084, \quad \alpha_{x}=0.613963 \\
& \beta_{y}=50.0533, \quad \alpha_{y}=-2.193219
\end{aligned}
$$

MAD Twiss output (Table 2)

$$
\begin{aligned}
& \beta_{x}=11.75939402, \quad \alpha_{x}=0.6635168614 \\
& \beta_{y}=54.09318824, \quad \alpha_{y}=-2.370235501 .
\end{aligned}
$$

From MAD Twiss couple output (Table 3 )

$$
\begin{aligned}
& \beta_{1}=17.82817229, \quad \alpha_{1}=1.890607154 \\
& \beta_{2}=120.7788642, \quad \alpha_{2}=-5.465936926 .
\end{aligned}
$$

There is large difference between the above three sets of Twiss parameters for the same lattice same initial conditions and none of thern coincides with the corresponding parameters of the projections of the 4-dimensional emittance ellipsoid on $x x^{\prime}$ and $y y^{\prime}$ plane. When the rotation angle $\xi$ is smaller, the discrepancy is smaller but they do not coincide with each other also. For example, if $\xi=0.0002735 \mathrm{rad}$, the results are shown as follows:

Projections on $x x^{\prime}$ and $y y^{\prime}$ planes

$$
\begin{aligned}
& \beta_{x}=11.78884012, \quad \alpha_{x}=0.663516342 \\
& \beta_{y}=54.0931771, \quad \alpha_{y}=-2.370234907 .
\end{aligned}
$$

SYNCH TRKB output

$$
\begin{aligned}
& \beta_{x}=11.7859, \quad \alpha_{x}=0.663354 \\
& \beta_{y}=54.07999, \quad \alpha_{y}=-2.369653 .
\end{aligned}
$$

MAD Twiss output

$$
\begin{aligned}
& \beta_{x}=11.78844069, \quad \alpha_{x}=0.6635168117 ; \\
& \beta_{y}=54.09318420, \quad \alpha_{y}=-2.370235323 .
\end{aligned}
$$

MAD Twiss couple output

$$
\begin{aligned}
& \beta_{1}=11.79540445, \quad \alpha_{1}=0.668622315 ; \\
& \beta_{2}=54.25392611, \quad \alpha_{2}=-2.377879573 .
\end{aligned}
$$


The discrepancy is clear even though the rotation is as small as $0.0002735 \mathrm{rad}$. This fact means that, in lattices with transverse coupling, in different programs the $\beta$ 's and $\alpha$ 's have different meaning and different value. One has to use these programs carefully in calculating or matching lattices with transverse coupling.

\section{Acknowledgments}

The author would like to thank Dr. J. Claus and Dr. H. Foelsche for their support and valuable discussions. 


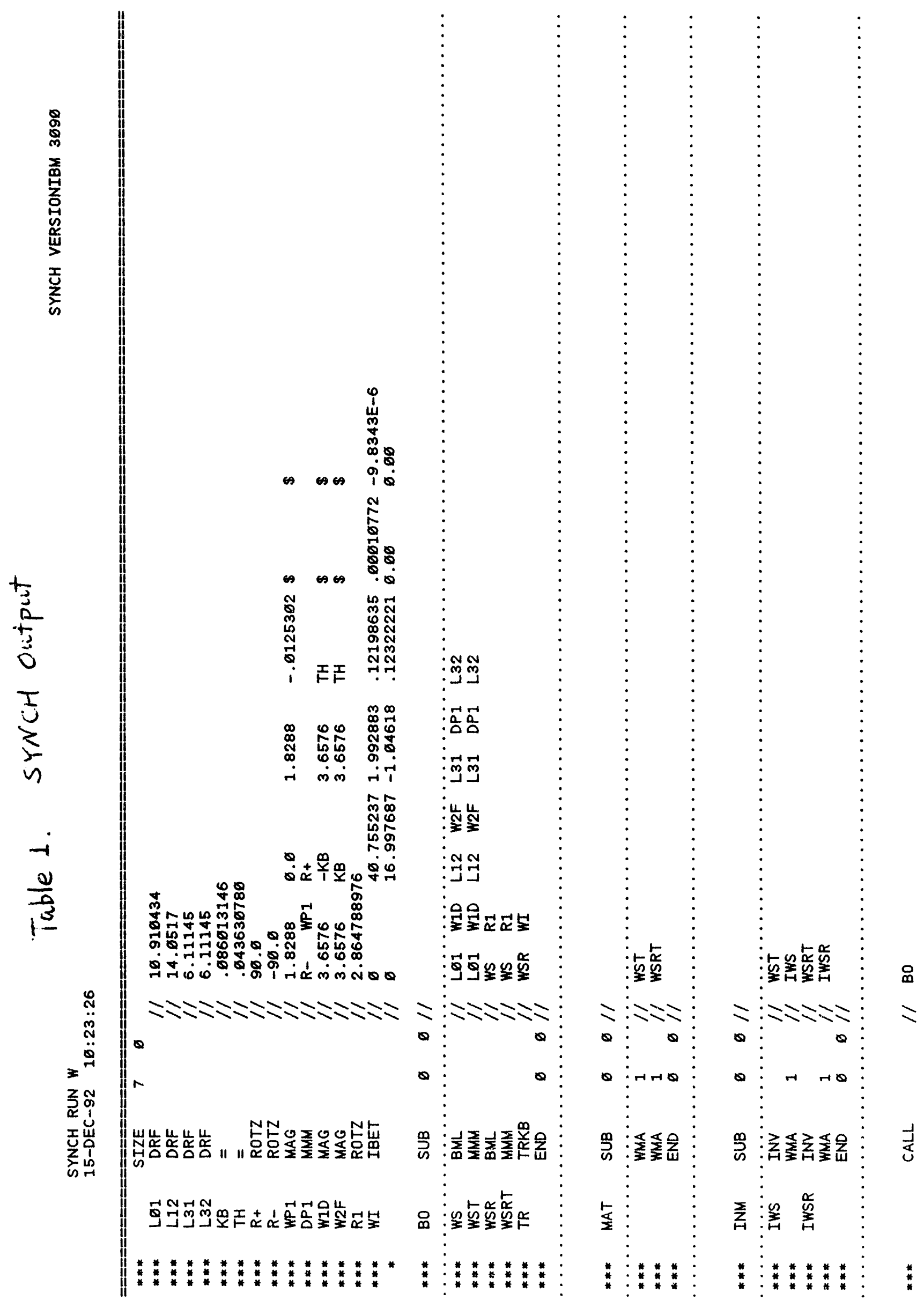




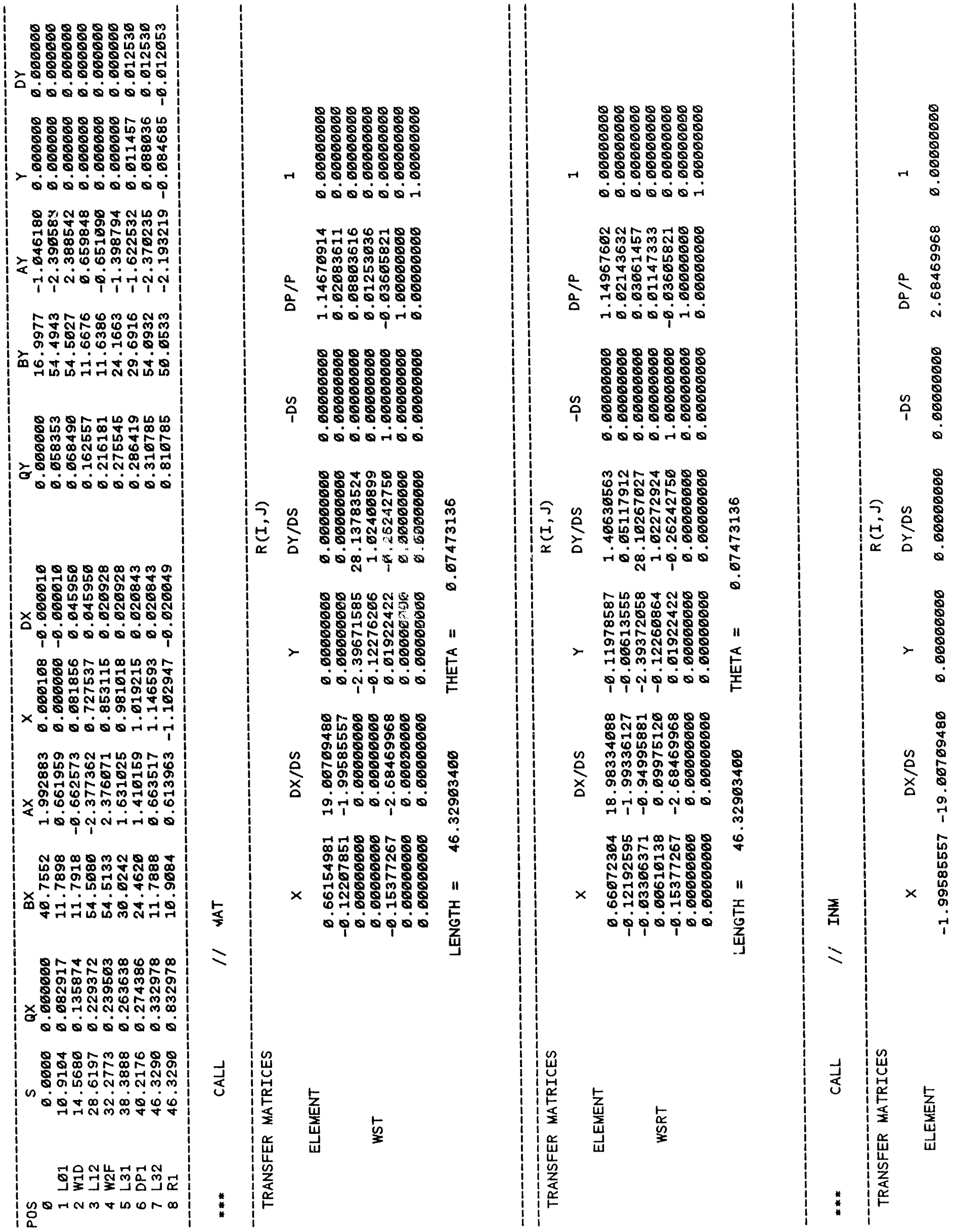




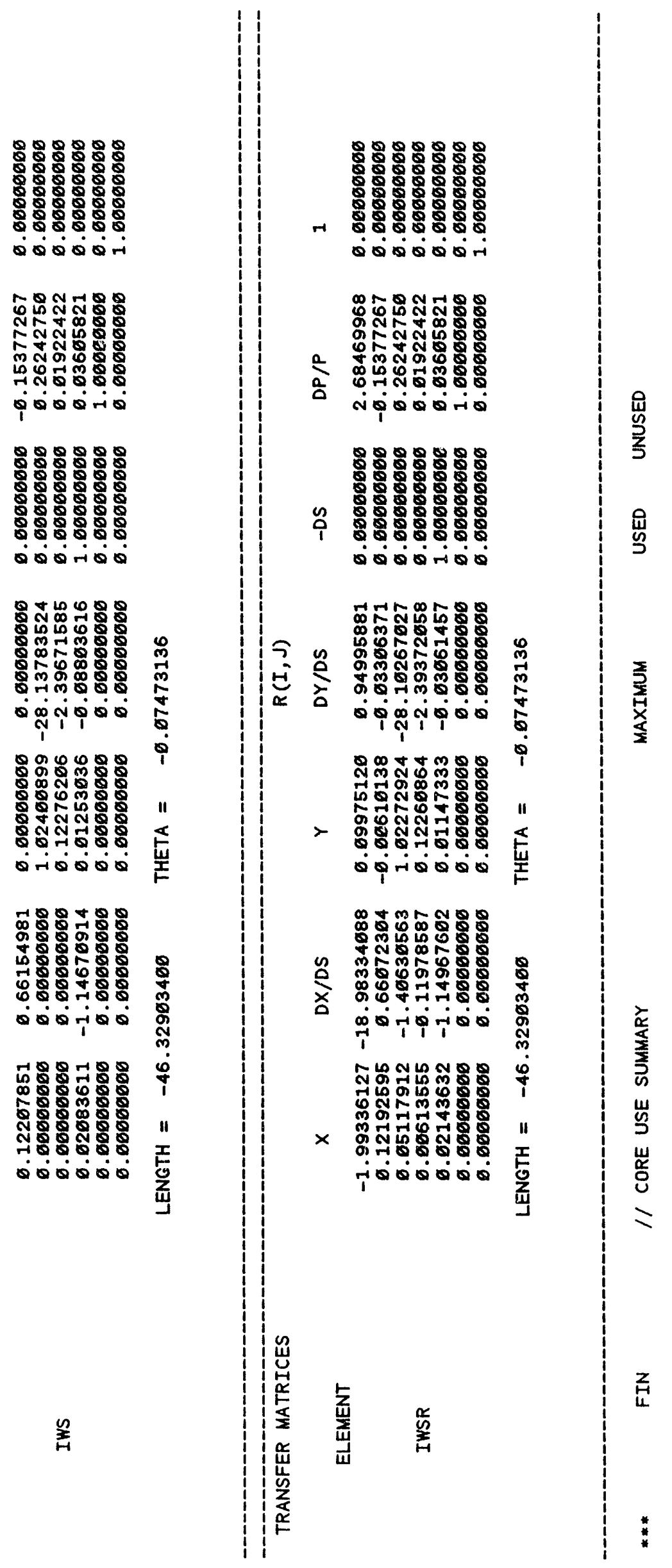




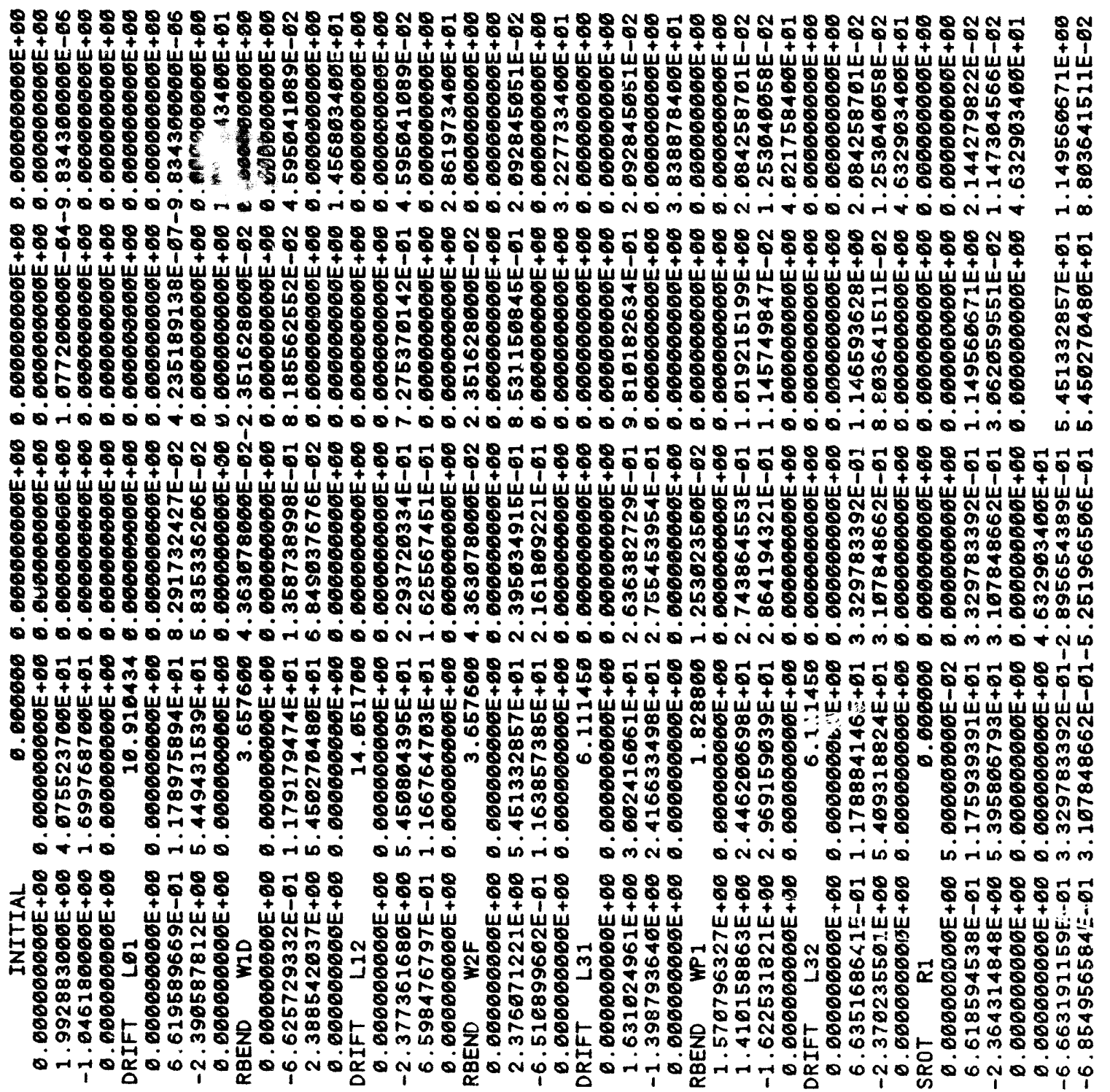


$\frac{0}{3}$

$\frac{n}{3}$

ì
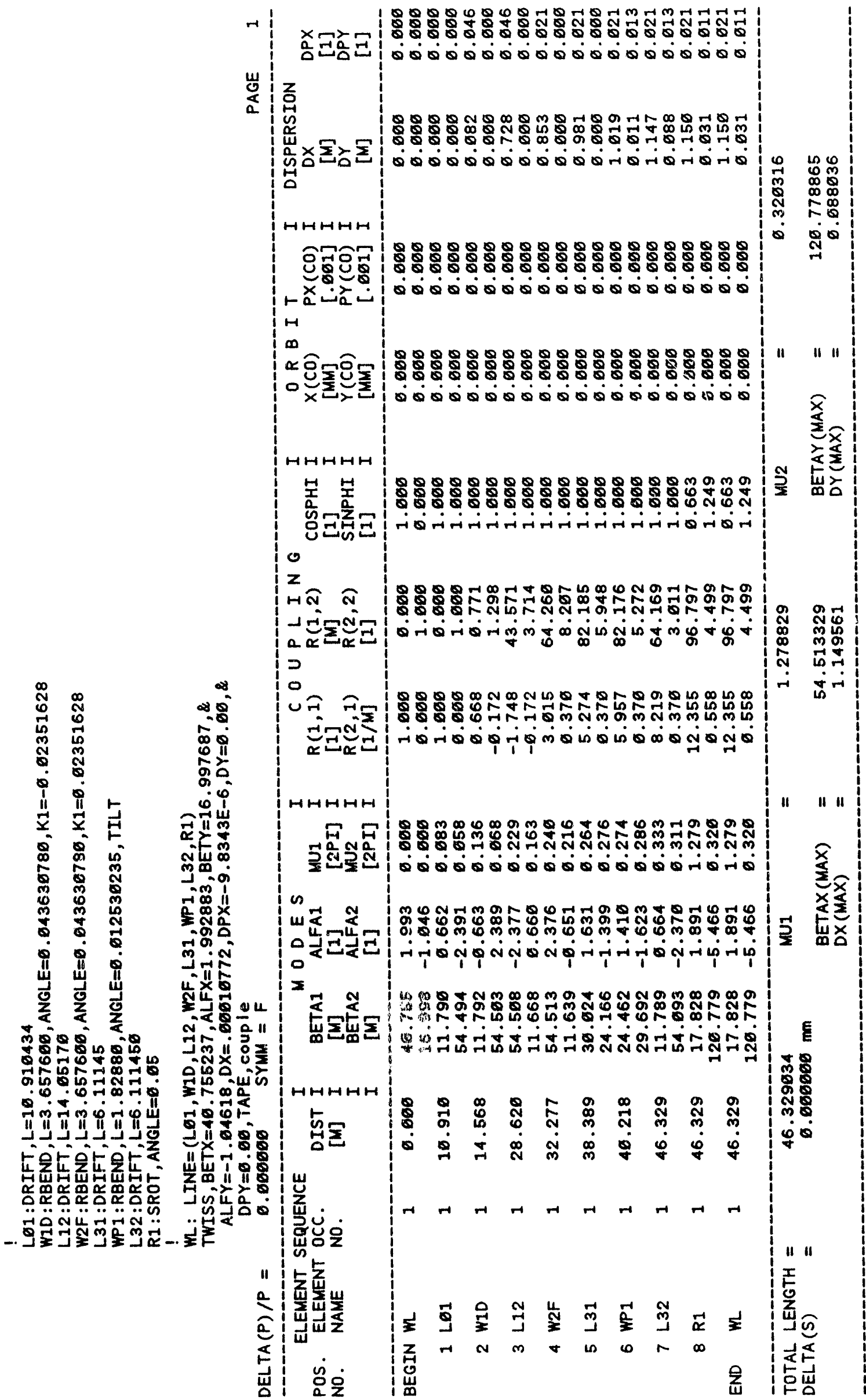


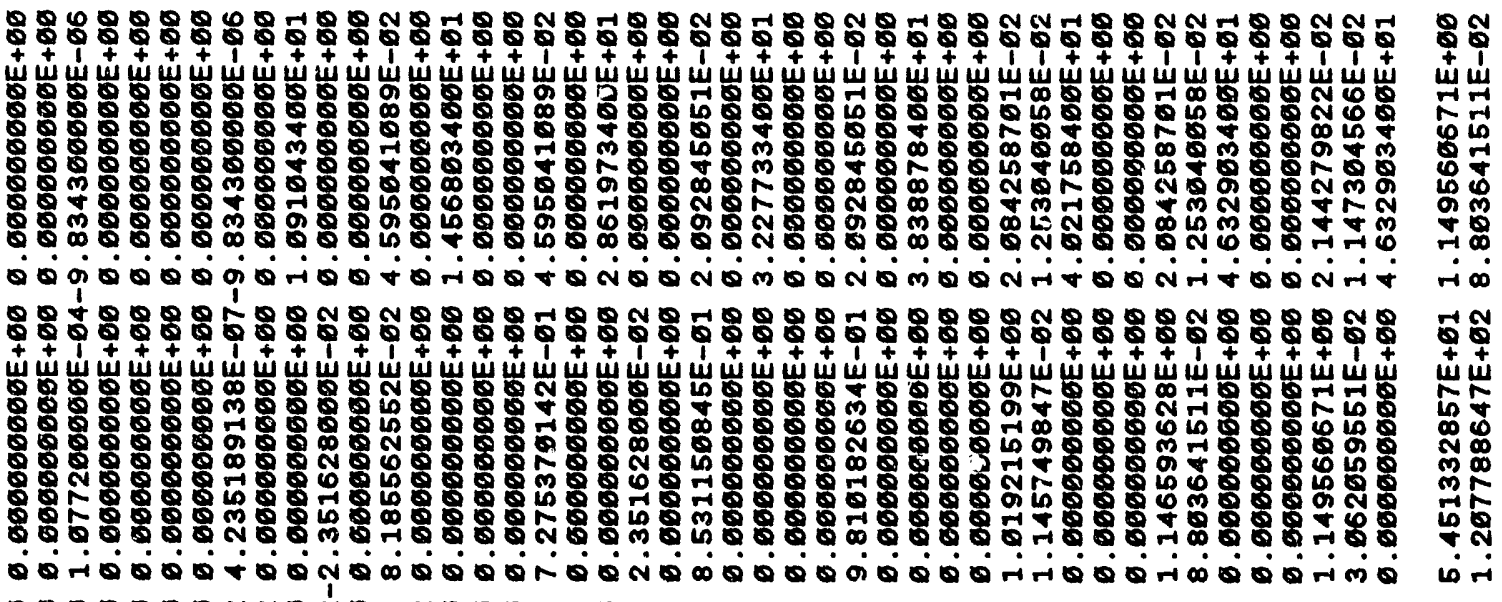

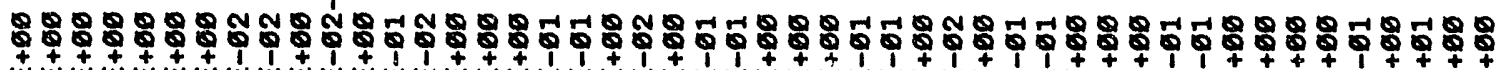

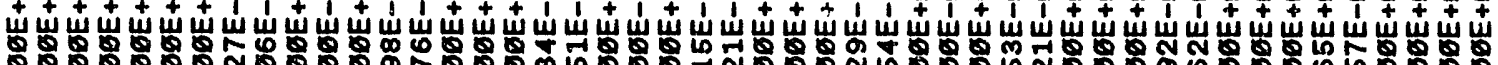

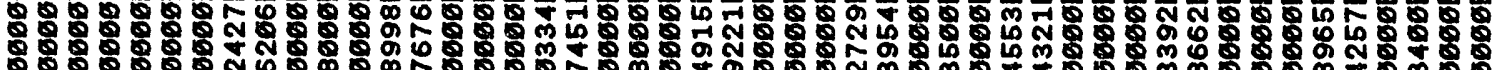

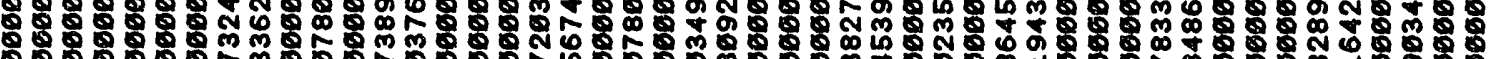

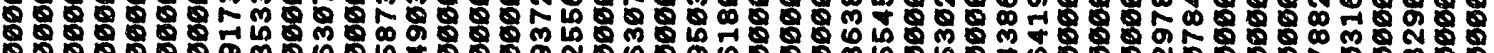

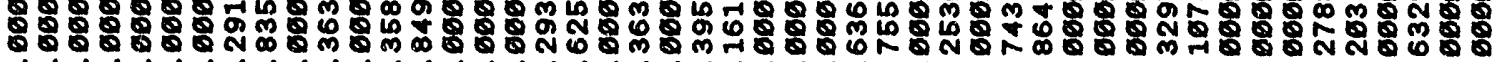

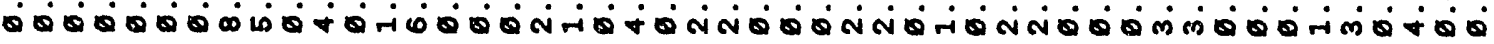

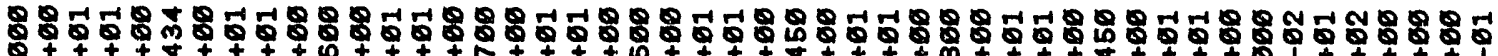

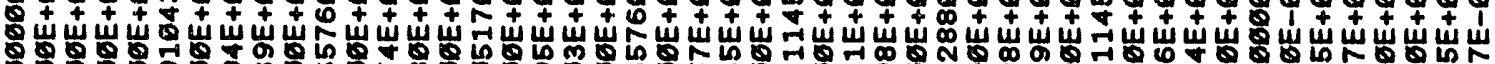

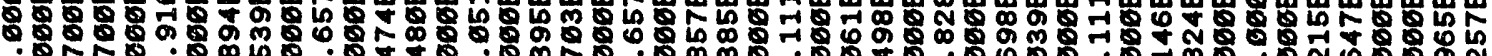
○告

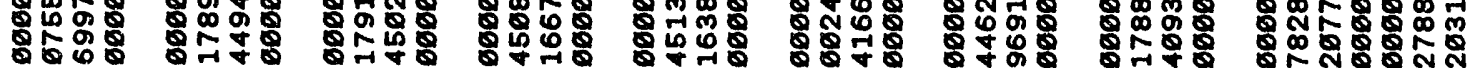

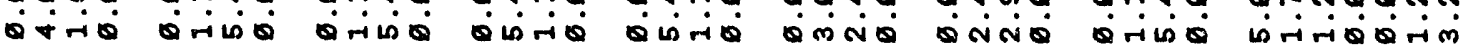

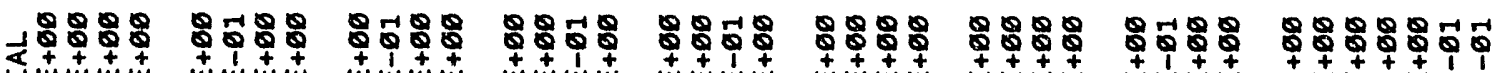

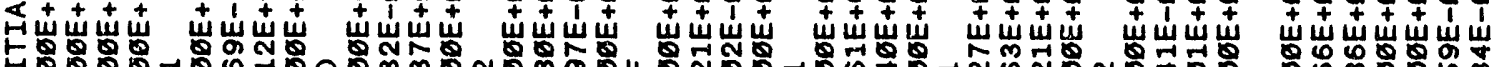

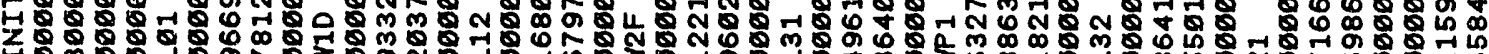

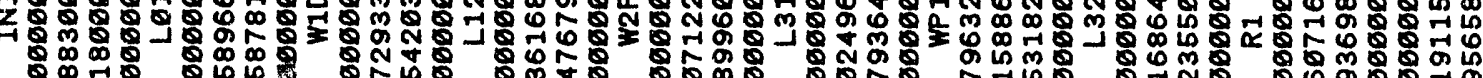
S. \& ब 

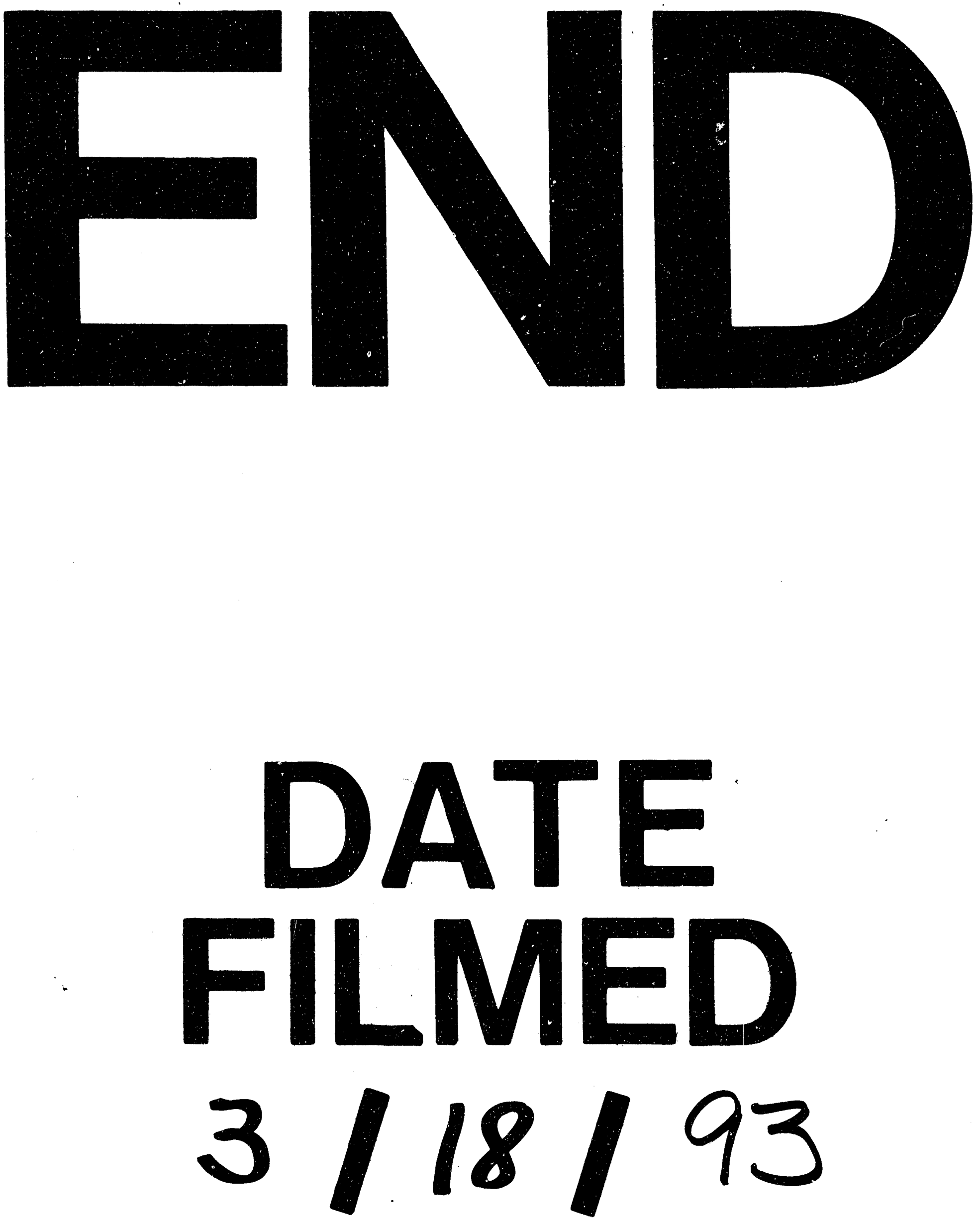
